\title{
A Note on the Acceleration and Jerk in Motion Along a Space Curve
}

\author{
Kahraman Esen Özen, Mehmet Güner and Murat Tosun
}

\begin{abstract}
The resolution of the acceleration vector of a particle moving along a space curve is well known thanks to Siacci [1]. This resolution comprises two special oblique components which lie in the osculating plane of the curve. The jerk is the time derivative of acceleration vector. For the jerk vector of the aforementioned particle, a similar resolution is presented as a new contribution to field [2]. It comprises three special oblique components which lie in the osculating and rectifying planes. In this paper, we have studied the Siacci's resolution of the acceleration vector and aforementioned resolution of the jerk vector for the space curves which are equipped with the modified orthogonal frame. Moreover, we have given some illustrative examples to show how the our theorems work.
\end{abstract}

\section{Introduction}

In Newtonian physics, it is well known that the force acting on a particle is concerned with its acceleration through the equation $\mathbf{F}=m \mathbf{a}$. A particle, which moves under the influence of arbitrary forces in 3-dimensional Euclidean space, has an acceleration which is obtained by the time derivative of the velocity vector, and thus by two time derivative of the position vector. For some applications, to state the acceleration vector as the sum of its tangential and

Key Words: Kinematics of a particle, Space curves, Siacci, Jerk, Modified orthogonal frame.

2010 Mathematics Subject Classification: Primary 70B05; Secondary 14H50.

Received: 07.04.2019

Accepted: 28.06.2019 
normal components is practical. However, when the angular momentum of the particle is constant, to state the acceleration vector as the sum of its tangential and radial components is more practical. The acceleration was stated in this form by Siacci in [1]. In Siacci's this study, the tangent component of acceleration lies along the tangent line of the trajectory while the radial component of acceleration lies in the instantaneous osculating plane to the trajectory [3]. In the literature, there have been numerous studies about the Siacci's theorem $[3,4,5,6,7]$.

The jerk vector is the time derivative of the acceleration vector. Thus, the equality $\mathbf{j}=\frac{1}{m} \frac{d \mathbf{F}}{d t}$ is satisfied for the particle which has a constant mass. Also, the jerk vector is different from the zero vector when the time derivative of the force is different from the zero vector. When a gymnast does gymnastic exercises or a stock-car racer races on track or a machinist drives a highspeed train, the acceleration changes suddenly. In these kind of situations, to estimate the lower threshold of just noticeable jerk and upper values of the jerk that can be tolerated by humans without undue discomfort is very important [8]. By Melchior in [9], these calculations were considered.

Due to Resal [10], the resolution of the jerk vector along a space curve in 3dimensional Euclidean space is well known. In this resolution, the jerk vector is obtained along the tangent, normal and binormal unit vectors of Serret-Frenet frame. This concept is still an issue of interest. Recently, a new decomposition of jerk vector along the tangential direction and radial directions in osculating and rectifying planes is presented in [2] by using the Serret- Frenet frame. This frame is a moving frame which has an important place in the theory of curves. It illustrates the characteristic properties of the curve since it provides the ability to ride along the curve.

Modified orthogonal frame was studied by Sasai in [11] for the curves whose curvature are not identically zero. There is a very close relation between this frame and Serret-Frenet frame. In the literature, there have been numerous studies about the Modified orthogonal frame [11, 12, 13, 14, 15].

In this paper, firstly, we have given a short knowledge about the Serret-Frenet frame and modified orthogonal frame. Afterwards, for a particle moving along an analytic space curve, which is equipped with the modified orthogonal frame, we have studied the Siacci's theorem by inspiring the study [3]. Also, we have obtained the resolution of the jerk vector along tangential and radial directions by inspiring the study [2]. Furthermore, two illustrative examples have been given to show how the our theorems work. 


\section{Preliminaries}

Let 3-dimensional Euclidean space $E^{3}$ be equipped with the standard inner product

$$
\langle\mathbf{A}, \mathbf{B}\rangle=a_{1} b_{1}+a_{2} b_{2}+a_{3} b_{3}
$$

where $\mathbf{A}=\left(a_{1}, a_{2}, a_{3}\right), \mathbf{B}=\left(b_{1}, b_{2}, b_{3}\right)$ are any vectors in $E^{3}$. The norm of a vector $\mathbf{A} \in E^{3}$ is defined as $\|\mathbf{A}\|=\sqrt{\langle\mathbf{A}, \mathbf{A}\rangle}$.

In $E^{3}$, a curve

$$
\begin{aligned}
\beta: J & \rightarrow E^{3} \\
t & \rightarrow \beta(t)=\left(\beta_{1}(t), \beta_{2}(t), \beta_{3}(t)\right)
\end{aligned}
$$

is called a differentiable curve if its coordinate functions

$$
\begin{aligned}
\beta_{i}: J & \rightarrow R \\
t & \rightarrow \beta_{i}(t), \quad i=1,2,3
\end{aligned}
$$

are differentiable in the non-empty open interval $J$ of the set of real numbers. If $\left(\beta_{1}{ }^{\prime}(t)\right)^{2}+\left(\beta_{2}{ }^{\prime}(t)\right)^{2}+\left(\beta_{3}{ }^{\prime}(t)\right)^{2} \neq 0$ for all $t \in J, \beta$ is called a non-singular curve. On the other hand, $\beta$ is a unit speed curve if $\left\|\beta^{\prime}(t)\right\|=1$ for all $t \in J$; in this case, $t$ is called arc-length parameter of the curve $\beta(t)$. Note that, all non-singular curves can be parameterized by its arc length.

Let a non-singular differentiable curve $\alpha$ be given as

$$
\begin{aligned}
\alpha: I & \rightarrow E^{3} \\
s & \rightarrow \alpha(s)=\left(\alpha_{1}(s), \alpha_{2}(s), \alpha_{3}(s)\right)
\end{aligned}
$$

by its arc length parameter $s$ where $I$ is a non-empty open interval of $R$. Suppose that $\alpha$ does not have vanishing second derivatives. Then, the moving Serret-Frenet frame of the aforementioned curve $\alpha(s)$ is well defined. It is denoted by $\{\mathbf{t}(s), \mathbf{n}(s), \mathbf{b}(s)\}$ where $\mathbf{t}(s), \mathbf{n}(s)$ and $\mathbf{b}(s)$ are the unit tangent, unit principal normal and unit binormal vectors respectively. The Serret-Frenet formulas are given as follows:

$$
\mathbf{t}^{\prime}=\kappa \mathbf{n}, \quad \mathbf{n}^{\prime}=-\kappa \mathbf{t}+\tau \mathbf{b}, \quad \mathbf{b}^{\prime}=-\tau \mathbf{n}
$$

where the curvature function $\kappa$ and the torsion function $\tau$ are defined by $\kappa=\kappa(s)=\left\|\mathbf{t}^{\prime}(s)\right\|$ and $\tau=\tau(s)=-\left\langle\mathbf{b}^{\prime}(s), \mathbf{n}(s)\right\rangle$, respectively [16].

Since the real functions $\alpha_{1}, \alpha_{2}$ and $\alpha_{3}$, which are given above, are differentiable in the open interval $I$, they are analytic in $I$, as well. Thus, the aforementioned 
differentiable curve $\alpha(s)$ is analytic in $I$. Then, for $\alpha$, there is an orthogonal frame $\{\mathbf{T}, \mathbf{N}, \mathbf{B}\}$ which is given as in the following:

$$
\mathbf{T}=\alpha^{\prime}(s), \mathbf{N}=\mathbf{T}^{\prime}(s), \mathbf{B}(s)=\mathbf{T}(s) \wedge \mathbf{N}(s)
$$

where $\wedge$ symbolizes the vector product. This frame is called modified orthogonal frame (see [11] for more details). The relations of this frame $\{\mathbf{T}, \mathbf{N}, \mathbf{B}\}$ and classical Serret-Frenet frame $\{\mathbf{t}, \mathbf{n}, \mathbf{b}\}$ are given as

$$
\mathbf{T}=\mathbf{t}, \mathbf{N}=\kappa \mathbf{n}, \mathbf{B}=\kappa \mathbf{b} .
$$

It can be seen easily that the lengths of the vectors $\mathbf{N}(s)$ and $\mathbf{B}(s)$ correspond to $\kappa(s)$. Due to the equation (3), after some calculations, the derivative formulas can be given as follows:

$$
\left(\begin{array}{l}
\mathbf{T}^{\prime} \\
\mathbf{N}^{\prime} \\
\mathbf{B}^{\prime}
\end{array}\right)=\left(\begin{array}{ccc}
0 & 1 & 0 \\
-\kappa^{2} & \frac{\kappa^{\prime}}{\kappa} & \tau \\
0 & -\tau & \frac{\kappa^{\prime}}{\kappa}
\end{array}\right)\left(\begin{array}{l}
\mathbf{T} \\
\mathbf{N} \\
\mathbf{B}
\end{array}\right)
$$

where

$$
\tau=\tau(s)=\frac{\operatorname{det}\left(\alpha^{\prime}, \alpha^{\prime \prime}, \alpha^{\prime \prime \prime}\right)}{\kappa^{2}}
$$

is the torsion of the curve $\alpha$. In the classical case, (4) corresponds to the Serret-Frenet equation. Furthermore, the equalities

$$
\langle\mathbf{T}, \mathbf{T}\rangle=1,\langle\mathbf{N}, \mathbf{N}\rangle=\langle\mathbf{B}, \mathbf{B}\rangle=\kappa^{2},\langle\mathbf{T}, \mathbf{N}\rangle=\langle\mathbf{T}, \mathbf{B}\rangle=\langle\mathbf{N}, \mathbf{B}\rangle=0
$$

are satisfied, [11].

Take into account of a particle of mass $m$ which travels along the aforementioned curve $\alpha(s)$ in $E^{3}$. Choose an arbitrary fixed origin $O$ in the space and denote by $\mathbf{x}$ the position vector of $P$ at time $t$. Here, the arc-length of $\alpha$ corresponds to the time $t$. The unit tangent vector for the curve $\alpha$ can be written as follows:

$$
\mathbf{T}=\frac{d \mathbf{x}}{d s} .
$$

Then, with the aid of (4) and (6), the velocity vector $\mathbf{v}$, the acceleration vector $\mathbf{a}$ and the jerk vector $\mathbf{J}$ of the particle at a time $t$ are obtained as in the following:

$$
\begin{aligned}
& \mathbf{v}=\frac{d \mathbf{x}}{d t}=\frac{d s}{d t} \mathbf{T} \\
& \mathbf{a}=\frac{d \mathbf{v}}{d t}=\frac{d^{2} s}{d t^{2}} \mathbf{T}+\left(\frac{d s}{d t}\right)^{2} \mathbf{N} \\
& \mathbf{J}=\left[\frac{d^{3} s}{d t^{3}}-\kappa^{2}\left(\frac{d s}{d t}\right)^{3}\right] \mathbf{T}+\left[3 \frac{d s}{d t} \frac{d^{2} s}{d t^{2}}+\frac{1}{\kappa} \frac{d \kappa}{d s}\left(\frac{d s}{d t}\right)^{3}\right] \mathbf{N}+\left[\tau\left(\frac{d s}{d t}\right)^{3}\right] \mathbf{B} .
\end{aligned}
$$




\section{Alternative Resolutions of Acceleration and Jerk Vec- tors According to Modified Orthogonal Frame}

A particle $P$, moving along the aforementioned curve $\alpha$, may be seen as a point of this curve. So, $P$ has a position vector according to the modified orthogonal frame. Let the position vector of $P$ be resolved as follows:

$$
\mathbf{x}=q \mathbf{T}-p \mathbf{N}+b \mathbf{B}
$$

where

$$
q=\langle\mathbf{x}, \mathbf{T}\rangle, \quad-p=\frac{1}{\kappa^{2}}\langle\mathbf{x}, \mathbf{N}\rangle, \quad b=\frac{1}{\kappa^{2}}\langle\mathbf{x}, \mathbf{B}\rangle .
$$

Denote by $\mathbf{r}$ and $\mathbf{r}^{*}$ the vectors

$$
\mathbf{r}=q \mathbf{T}-p \mathbf{N}, \quad \mathbf{r}^{*}=q \mathbf{T}+b \mathbf{B}
$$

which lie in the osculating plane $\pi_{1}$ to $C$ at $P$ and in the rectifying plane $\pi_{2}$ to $C$ at $P$, respectively. In that case, we get

$$
r^{2}=\langle\mathbf{r}, \mathbf{r}\rangle=q^{2}+\kappa^{2} p^{2}, \quad\left(r^{*}\right)^{2}=\left\langle\mathbf{r}^{*}, \mathbf{r}^{*}\right\rangle=q^{2}+\kappa^{2} b^{2}
$$

where $r$ and $r^{*}$ are the lengths of the vectors $\mathbf{r}$ and $\mathbf{r}^{*}$, respectively. (See Figure 1).

By vector multiplication of the position vector $\mathbf{x}$, aforementioned in (8), and the linear momentum vector $m\left(\frac{d s}{d t}\right) \mathbf{T}$, the angular momentum vector of $P$ about $O$ is obtained as

$$
\mathbf{H}^{O}=m b\left(\frac{d s}{d t}\right) \mathbf{N}+m p\left(\frac{d s}{d t}\right) \mathbf{B}
$$

By following the similar steps in the studies [3] and [2], now we try to resolve the acceleration vector $\mathbf{a}$ in (7) along the radial direction $B P$ and tangential direction in osculating plane $\pi_{1}$, and also try to resolve the jerk vector $\mathbf{J}$ in (7) along the tangential direction, radial direction $B P$ in osculating plane $\pi_{1}$ and radial direction $Y P$ in the rectifying plane $\pi_{2}$. To do so, firstly, let us express the vector $\mathbf{N}$ in terms of $\mathbf{r}$ and $\mathbf{T}$. In view of (10), we can conclude that this is possible if and only if $p \neq 0$. By making the physical assumption that the component of angular momentum along the vector $\mathbf{B}$ never vanishes, we can ensure that $p$ is nonzero. Secondly, let us express the vector $\mathbf{B}$ in terms of $\mathbf{r}^{*}$ and $\mathbf{T}$. From the perspective of equation (10), this is possible if and only if $b \neq 0$. By making the second physical assumption that the component of angular momentum along the vector $\mathbf{N}$ never vanishes, we can ensure that $b$ is 


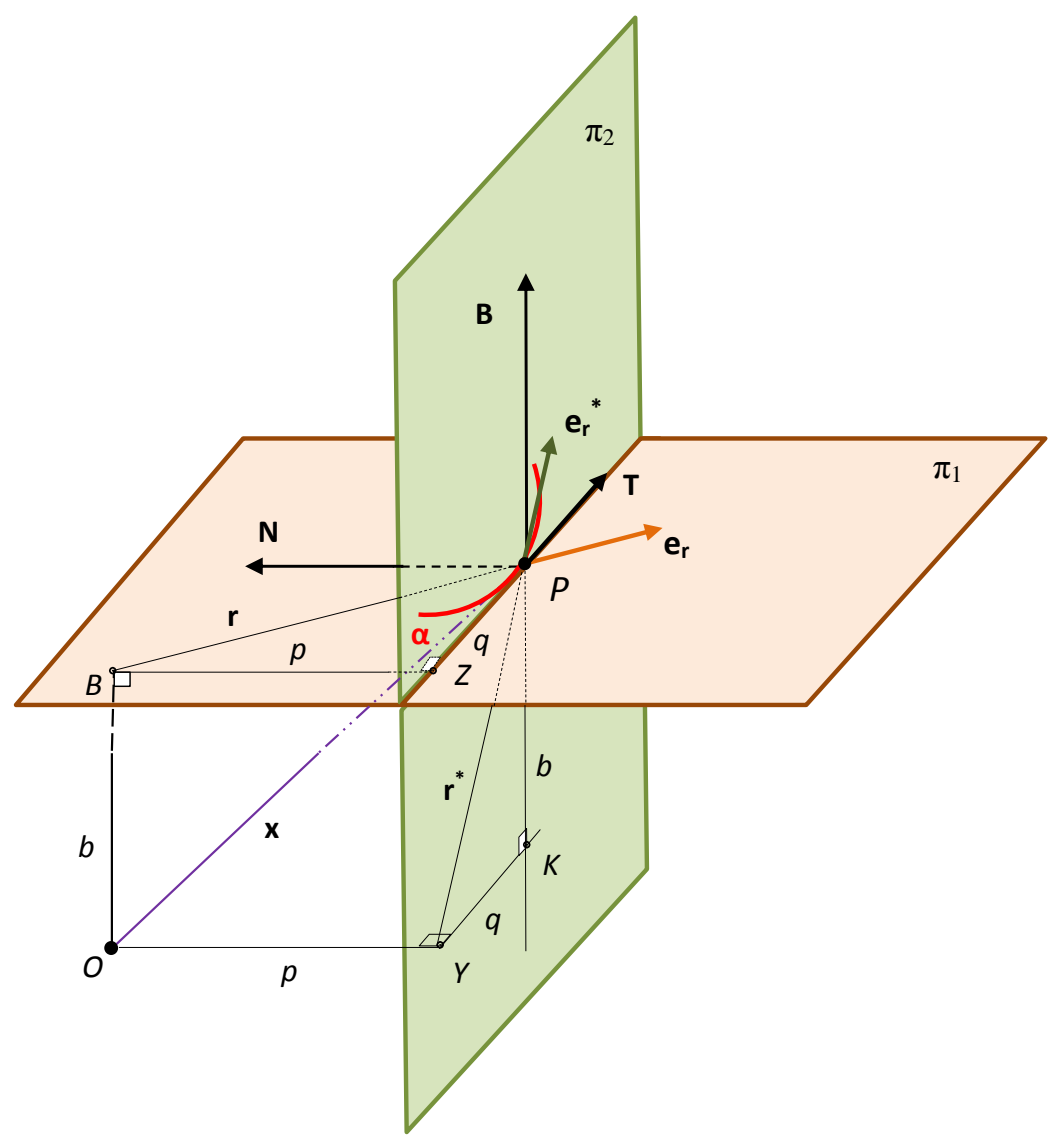

Figure 1: A particle $P$ moves on an analytic curve $\alpha$ in the 3D Euclidean space $E^{3} . \pi_{1}$ and $\pi_{2}$ are the osculating and rectifying planes at $P$, respectively. $B$ is the foot of the perpendicular line segment which is from the origin $O$ to the plane $\pi_{1} . Y$ is the foot of the perpendicular line segment which is from the origin to the plane $\pi_{2} . B Z$ and $Y K$ are perpendicular line segments to the tangent and binormal axes, respectively. $\mathbf{r}$ and $\mathbf{r}^{*}$ are the position vectors of $P$ relative to $B$ and $Y$. $\mathbf{e}_{\mathbf{r}}$ is the unit vector in the direction of $B P$ and $\mathbf{e}_{\mathbf{r}}{ }^{*}$ is the unit vector in the direction of $Y P$. 
nonzero. Taking into consideration these assumptions, the following equations can be written:

$$
\mathbf{N}=\frac{1}{p}(-\mathbf{r}+q \mathbf{T}), \quad \mathbf{B}=\frac{1}{b}\left(-q \mathbf{T}+\mathbf{r}^{*}\right) .
$$

From the equation (11) and the aforementioned physical assumptions, $r \neq 0$ and $r^{*} \neq 0$. Thus, the unit vectors $\mathbf{e}_{\mathbf{r}}$ and $\mathbf{e}_{\mathbf{r}}{ }^{*}$ can be defined as

$$
\mathbf{e}_{\mathbf{r}}=\frac{1}{r} \mathbf{r}, \quad \mathbf{e}_{\mathbf{r}}^{*}=\frac{1}{r^{*}} \mathbf{r}^{*} .
$$

By means of (13) and (14), we get

$$
\mathbf{N}=\frac{1}{p}\left(-r \mathbf{e}_{\mathbf{r}}+q \mathbf{T}\right), \quad \mathbf{B}=\frac{1}{b}\left(-q \mathbf{T}+r^{*} \mathbf{e}_{\mathbf{r}}{ }^{*}\right) .
$$

Now, if we substitute the equations (15) into the equation (7), we obtain the acceleration vector $\mathbf{a}$ and jerk vector $\mathbf{J}$ as

$$
\mathbf{a}=\left[\frac{d^{2} s}{d t^{2}}+\frac{q}{p}\left(\frac{d s}{d t}\right)^{2}\right] \mathbf{T}+\left[-\frac{r}{p}\left(\frac{d s}{d t}\right)^{2}\right] \mathbf{e}_{\mathbf{r}}=S_{t} \mathbf{T}+S_{r} \mathbf{e}_{\mathbf{r}}
$$

and

$$
\begin{aligned}
\mathbf{J} & =\left[\frac{d^{3} s}{d t^{3}}-\kappa^{2}\left(\frac{d s}{d t}\right)^{3}+3 \frac{q}{p} \frac{d s}{d t} \frac{d^{2} s}{d t^{2}}+\frac{q}{p \kappa} \frac{d \kappa}{d s}\left(\frac{d s}{d t}\right)^{3}-\frac{q \tau}{b}\left(\frac{d s}{d t}\right)^{3}\right] \mathbf{T} \\
& +\left[-3 \frac{r}{p} \frac{d s}{d t} \frac{d^{2} s}{d t^{2}}-\frac{r}{p \kappa} \frac{d \kappa}{d s}\left(\frac{d s}{d t}\right)^{3}\right] \mathbf{e}_{\mathbf{r}} \\
& +\left[\frac{r^{*} \tau}{b}\left(\frac{d s}{d t}\right)^{3}\right] \mathbf{e}_{\mathbf{r}}^{*} \\
& =T_{t} \mathbf{T}+T_{r} \mathbf{e}_{\mathbf{r}}+T_{r^{*}} \mathbf{e}_{\mathbf{r}}{ }^{*} .
\end{aligned}
$$

Here, $S_{t}, S_{r}$ are tangential and radial Siacci components of the acceleration, while $T_{t}, T_{r}, T_{r^{*}}$ are tangential and radial components of the jerk.

Taking into consideration the above derivation about the acceleration and jerk vectors of the particle $P$, the following theorems can be given.

Theorem 1 (Siacci's Theorem According to Modified Orthogonal Frame). Let $P$ be a particle whose mass is $m$ and which moves along an analytic space curve $\alpha$ equipped with the modified orthogonal frame. Assume that the component of 
its angular momentum which is along the vector $\mathbf{B}$ never takes the value zero. In this case, the acceleration vector $\mathbf{a}$ of $P$ could be stated as

$$
\mathbf{a}=\left[\frac{d^{2} s}{d t^{2}}+\frac{q}{p}\left(\frac{d s}{d t}\right)^{2}\right] \mathbf{T}+\left[-\frac{r}{p}\left(\frac{d s}{d t}\right)^{2}\right] \mathbf{e}_{\mathbf{r}}=S_{t} \mathbf{T}+S_{r} \mathbf{e}_{\mathbf{r}}
$$

$S_{r}$ is directed from the particle(point) $P$ towards the foot of the perpendicular that is from the origin to the osculating plane $\pi_{1}$ to $\alpha$ at $P$, while $S_{t}$ lies along the tangent line of $\alpha$.

Theorem 2. Let $P$ be a particle whose mass is $m$ and which moves along an analytic space curve $\alpha$ equipped with the modified orthogonal frame. Assume that each of the components of its angular momentum vector never vanishes. In this case, the jerk vector $\mathbf{J}$ of $P$ could be stated as:

$$
\begin{aligned}
\mathbf{J} & =\left[\frac{d^{3} s}{d t^{3}}-\kappa^{2}\left(\frac{d s}{d t}\right)^{3}+3 \frac{q}{p} \frac{d s}{d t} \frac{d^{2} s}{d t^{2}}+\frac{q}{p \kappa} \frac{d \kappa}{d s}\left(\frac{d s}{d t}\right)^{3}-\frac{q \tau}{b}\left(\frac{d s}{d t}\right)^{3}\right] \mathbf{T} \\
& +\left[-3 \frac{r}{p} \frac{d s}{d t} \frac{d^{2} s}{d t^{2}}-\frac{r}{p \kappa} \frac{d \kappa}{d s}\left(\frac{d s}{d t}\right)^{3}\right] \mathbf{e}_{\mathbf{r}} \\
& +\left[\frac{r^{*} \tau}{b}\left(\frac{d s}{d t}\right)^{3}\right] \mathbf{e}_{\mathbf{r}}^{*} \\
& =T_{t} \mathbf{T}+T_{r} \mathbf{e}_{\mathbf{r}}+T_{r^{*}} \mathbf{e}_{\mathbf{r}}^{*} .
\end{aligned}
$$

$T_{t}, T_{r}, T_{r^{*}}$ are tangential and radial components of the jerk. The component $T_{t}$ lies along the tangent line of $\alpha$. The component $T_{r}$ lies along the line that passes through the particle $P$ and the foot of the perpendicular which is from the origin to the osculating plane. The component $T_{r^{*}}$ lies along the line that passes through the particle $P$ and the foot of the perpendicular which is from the origin to the rectifying plane.

Corollary 1. In Euclidean 3-space, let the particle $P$ move along an analytic space curve which lies in the osculating plane $\pi_{1}$. Assume that the component of its angular momentum vector along the normal vector of the motion plane never vanishes. In that case, tangential component of the jerk vector $\mathbf{J}$ reduces to

$$
T_{t}=\frac{d^{3} s}{d t^{3}}-\kappa^{2}\left(\frac{d s}{d t}\right)^{3}+3 \frac{q}{p} \frac{d s}{d t} \frac{d^{2} s}{d t^{2}}+\frac{q}{p \kappa} \frac{d \kappa}{d s}\left(\frac{d s}{d t}\right)^{3} .
$$

The second radial component $T_{r^{*}}$ vanishes, while the first radial component does not change.

Proof. The proof can be completed easily by considering $\tau=0$ for the planar case. 


\section{Applications}

In this section, we give illustrative examples to show how the our theories work.

Example 1. Suppose that a particle $P$ travels along a right handed circular helix lying on a cylinder of radius $R$ and that the angular frequency $\omega$ of $P$ is not time dependent. In that case, the position vector of $P$ is as in the following:

$$
\mathbf{x}=R \cos (\omega t) \mathbf{i}+R \sin (\omega t) \mathbf{j}+B t \mathbf{k}
$$

where $t$ states the time. Here, $B$ is a positive constant and $\{\mathbf{i}, \mathbf{j}, \mathbf{k}\}$ is a fixed right-handed orthonormal frame. Let $\mathbf{k}$ be the axis of the helix and $\alpha$ be the helix angle satisfying the equality $\tan \alpha=\frac{R \omega}{B}$. The velocity vector, acceleration vector and jerk vector of $P$ could be calculated as follows:

$$
\begin{aligned}
& \mathbf{v}=-R \omega \sin (\omega t) \mathbf{i}+R \omega \cos (\omega t) \mathbf{j}+B \mathbf{k}, \\
& \mathbf{a}=-R \omega^{2} \cos (\omega t) \mathbf{i}-R \omega^{2} \sin (\omega t) \mathbf{j}, \\
& \mathbf{J}=R \omega^{3} \sin (\omega t) \mathbf{i}-R \omega^{3} \cos (\omega t) \mathbf{j} .
\end{aligned}
$$

From here, we can write the followings

$$
d x=-R \omega \sin (\omega t) d t, \quad d y=R \omega \cos (\omega t) d t, \quad d z=B d t, \quad d s=\lambda d t .
$$

where $\lambda=\sqrt{R^{2} \omega^{2}+B^{2}}$. Therefore,

$$
\frac{d s}{d t}=\lambda, \quad \frac{d^{2} s}{d t^{2}}=0, \quad \frac{d^{3} s}{d t^{3}}=0 .
$$

It is not difficult to see that the oriented curve which is traced out by the particle $P$ can be parameterized by the arc-length $s=s(t)=\lambda t$ as follows:

$$
\gamma(s)=\left(R \cos \left(\frac{\omega s}{\lambda}\right), R \sin \left(\frac{\omega s}{\lambda}\right), \frac{B s}{\lambda}\right) .
$$

Considering (2), the vectors $\mathbf{T}, \mathbf{N}$ and $\mathbf{B}$ for the helix can be obtained as

$$
\begin{aligned}
& \mathbf{T}=-\sin \alpha \sin \left(\frac{\omega s}{\lambda}\right) \mathbf{i}+\sin \alpha \cos \left(\frac{\omega s}{\lambda}\right) \mathbf{j}+\cos \alpha \mathbf{k} \\
& \mathbf{N}=-\frac{\sin ^{2} \alpha}{R} \cos \left(\frac{\omega s}{\lambda}\right) \mathbf{i}-\frac{\sin ^{2} \alpha}{R} \sin \left(\frac{\omega s}{\lambda}\right) \mathbf{j} \\
& \mathbf{B}=\frac{\sin ^{2} \alpha}{R} \cos \alpha \sin \left(\frac{\omega s}{\lambda}\right) \mathbf{i}-\frac{\sin ^{2} \alpha}{R} \cos \alpha \cos \left(\frac{\omega s}{\lambda}\right) \mathbf{j}+\frac{\sin ^{3} \alpha}{R} \mathbf{k} .
\end{aligned}
$$


As can be seen easily, the curvature $\kappa$ and the torsion $\tau$ are constant:

$$
\kappa=\frac{R \omega^{2}}{\lambda^{2}}, \quad \tau=\frac{B \omega}{\lambda^{2}} .
$$

From (9), (18), (19) and (20), we can obtain

$$
q=t B \cos \alpha, \quad p=\frac{R^{2}}{\sin ^{2} \alpha}, \quad b=\frac{t B R}{\sin \alpha} .
$$

On the other hand, the equalities

$$
r=\sqrt{R^{2}+t^{2} B^{2} \cos ^{2} \alpha}, \quad r^{*}=t B
$$

can be written in view of (11), (20) and (21).

By applying Theorem 1, we obtain the tangential and radial Siacci components of acceleration vector as

$$
S_{t}=\frac{t \lambda^{2} B \cos \alpha \sin ^{2} \alpha}{R^{2}}, \quad S_{r}=-\frac{\lambda^{2} \sin ^{2} \alpha \sqrt{R^{2}+t^{2} B^{2} \cos ^{2} \alpha}}{R^{2}} .
$$

Similarly, by applying Theorem 2, we obtain the tangential and radial components of jerk vector as

$$
T_{t}=-\frac{R^{2} \omega^{4}}{\lambda}-\frac{\lambda \omega B \cos \alpha \sin \alpha}{R}, \quad T_{r}=0, \quad T_{r^{*}}=\frac{\lambda B \omega \sin \alpha}{R} .
$$

Example 2. Suppose that a particle $P$ moves along the logarithmic spiral curve

$$
\delta(t)=\left(e^{\omega t} \cos (\omega t), 0, e^{\omega t} \sin (\omega t)\right)
$$

in $E^{3}$. Then, the position vector of $P$ is as in the following:

$$
\mathbf{x}=\left(e^{\omega t} \cos (\omega t), 0, e^{\omega t} \sin (\omega t)\right)
$$

where $t$ and $\omega$ symbolize time and angular frequency, respectively. The velocity, acceleration and jerk vectors concerned with the particle $P$ are obtained easily as follows:

$$
\begin{aligned}
& \mathbf{v}=\omega\left(e^{\omega t} \cos (\omega t)-e^{\omega t} \sin (\omega t), 0, e^{\omega t} \sin (\omega t)+e^{\omega t} \cos (\omega t)\right), \\
& \mathbf{a}=2 \omega^{2}\left(-e^{\omega t} \sin (\omega t), 0, e^{\omega t} \cos (\omega t)\right), \\
& \mathbf{J}=2 \omega^{3}\left(-e^{\omega t} \sin (\omega t)-e^{\omega t} \cos (\omega t), 0, e^{\omega t} \cos (\omega t)-e^{\omega t} \sin (\omega t)\right) .
\end{aligned}
$$

The equalities 


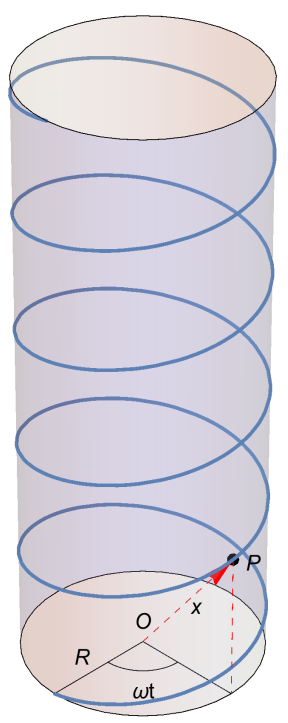

Figure 2: The right-handed circular helix on a cylinder of radius $R$ as the path of the particle $P$ which has an angular frequency $\omega$ ( $\omega$ is not time dependent).

$$
d x=\omega e^{\omega t}(\cos (\omega t)-\sin (\omega t)) d t, \quad d z=\omega e^{\omega t}(\sin (\omega t)+\cos (\omega t)) d t
$$

can be seen easily. Then, we get

$$
\frac{d s}{d t}=\sqrt{2} \omega e^{\omega t}, \quad \frac{d^{2} s}{d t^{2}}=\sqrt{2} \omega^{2} e^{\omega t}, \quad \frac{d^{3} s}{d t^{3}}=\sqrt{2} \omega^{3} e^{\omega t}
$$

with the aid of the equality $(d s)^{2}=(d x)^{2}+(d y)^{2}+(d z)^{2}$. It is not difficult to see that the oriented curve, traced out by the particle, can be reparameterized by the arc-length $s=s(t)=\sqrt{2} e^{\omega t}-\sqrt{2}$ as follows:

$$
\delta^{*}(s)=\left(\frac{s+\sqrt{2}}{\sqrt{2}} \cos \ln \left(\frac{s+\sqrt{2}}{\sqrt{2}}\right), 0, \frac{s+\sqrt{2}}{\sqrt{2}} \sin \ln \left(\frac{s+\sqrt{2}}{\sqrt{2}}\right)\right) .
$$

If we use the equations (2) and (28) we can easily find the Modified orthogonal frame vectors: 


$$
\begin{aligned}
& \mathbf{T}=\left(\frac{\cos \ln \left(\frac{s+\sqrt{2}}{\sqrt{2}}\right)-\sin \ln \left(\frac{s+\sqrt{2}}{\sqrt{2}}\right)}{\sqrt{2}}, 0, \frac{\cos \ln \left(\frac{s+\sqrt{2}}{\sqrt{2}}\right)+\sin \ln \left(\frac{s+\sqrt{2}}{\sqrt{2}}\right)}{\sqrt{2}}\right) \\
& \mathbf{N}=\left(\frac{-\cos \ln \left(\frac{s+\sqrt{2}}{\sqrt{2}}\right)-\sin \ln \left(\frac{s+\sqrt{2}}{\sqrt{2}}\right)}{\sqrt{2}(s+\sqrt{2})}, 0, \frac{\cos \ln \left(\frac{s+\sqrt{2}}{\sqrt{2}}\right)-\sin \ln \left(\frac{s+\sqrt{2}}{\sqrt{2}}\right)}{\sqrt{2}(s+\sqrt{2})}\right) \\
& \mathbf{B}=\left(0, \frac{1}{\sqrt{2}(s+\sqrt{2})}, 0\right)
\end{aligned}
$$

The curvature and torsion are constant:

$$
\kappa=\frac{1}{s+\sqrt{2}}, \quad \tau=0 .
$$

From this last equation, (9), (11), (28) and (29) we obtain:

$$
q=\frac{s+\sqrt{2}}{2}, p=\frac{(s+\sqrt{2})^{2}}{2}, b=0, r=\frac{s+\sqrt{2}}{\sqrt{2}}, r^{*}=\frac{s+\sqrt{2}}{2} .
$$

The acceleration vector is obtained for the particle $P$ in terms of tangential and radial components as

$$
\mathbf{a}=\left(2 \sqrt{2} \omega^{2} e^{\omega t}\right) \mathbf{T}+\left(-2 \sqrt{2} \omega^{2} e^{2 \omega t}\right) \mathbf{e}_{r}
$$

by applying Theorem 1. Similarly, the jerk vector is obtained in terms of tangential and radial components as

$$
\mathbf{J}=\left(2 \sqrt{2} \omega^{3} e^{\omega t}\right) \mathbf{T}+\left(-4 \omega^{3} e^{\omega t}\right) \mathbf{e}_{r}
$$

by applying Corollary 1.

\section{References}

[1] F. Siacci, Moto per Una Linea Gobba. Atti R Accad Sci. Torino 14 (1879), 946-951.

[2] K. E. Özen, F. S. Dündar, M. Tosun, An Alternative Approach to Jerk in Motion Along a Space Curve with Applications. Journal of Theoretical and Applied Mechanics 57(2) (2019), 435-444. 
[3] J. Casey, Siacci's Resolution of the Acceleration Vector for a Space Curve. Meccanica 46 (2011), 471-476.

[4] E. T. Whittaker, A Treatise on the Analytical Dynamics of Particles and Rigid Bodies. 4th Edition, Cambridge University Press, 1944.

[5] N. Grossman, The Sheer Joy of Celestial Mechanics. Birkhauser, Basel, 1996.

[6] Z. KüçüKarslan, M. Y. Yilmaz, M. Bektaş, Siacci's Theorem for Curves in Finsler Manifold $\mathbf{F}^{\mathbf{3}}$. Turkish Journial of Science and Technology $\mathbf{7}(\mathbf{2})$ (2012), 181-185.

[7] K. E. Özen, M. Tosun, M. Akyıãıt, Siacci's Theorem According to Darboux Frame. An. St. Univ. Ovidius Constanta 25(3) (2017), 155-165.

[8] S. H. Schot, Jerk: The Time Rate of Change of Acceleration. American Journal of Physics 46(11) (1978), 1090-1094.

[9] P. Melchior, Zeitschrift Ver. Dtsch. Ing. 72 (1928), 1842.

[10] H. Resal, Traite de Cinematique Pure. Mallet-Bachelier, 1862.

[11] T. Sasai, The Fundamental Theorem of Analytic Space Curves and Apparent Singularities of Fuchsian Differential Equations. Tohoku Math. Journ. 36 (1984), 17-24.

[12] B. Bukcu, M. K. Karacan, On the Modified Orthogonal Frame with Curvature and Torsion in 3-space. Mathematical Sciences and Applications E-Notes 4(1) (2016) 184-188.

[13] B. Bukcu, M. K. Karacan, Spherical Curves with Modified Orthogonal Frame. Journal of New Results in Science 5(10) (2016) 60-68.

[14] M. S. Lone, E. S. Hasan, M. K. Karacan, B. Bukcu, On Some Curves with Modified Orthogonal Frame in Euclidean 3-space. Iranian Journal of Science and Technology, Transactions A: Science (2018) 1-12.

[15] M. S. Lone, E. S. Hasan, M. K. Karacan, B. Bukcu, Mannheim Curves with Modified Orthogonal Frame in Euclidean 3-space. Turkish Journal of Mathematics 43(2) (2019), 648-663.

[16] T. Shifrin, Differential Geometry: A First Course in Curves and Surfaces. University of Georgia, Preliminary Version, 2008. 
Kahraman Esen ÖZEN,

Turkey.

Email: kesenozen@gmail.com

Mehmet GÜNER,

Department of Mathematics,

Sakarya University,

54187 Sakarya, Turkey.

Email: mguner@sakarya.edu.tr

Murat TOSUN,

Department of Mathematics,

Sakarya University,

54187 Sakarya, Turkey.

Email: tosun@sakarya.edu.tr 